Байкальский государственный университет, г. Иркутск, Российская Федерация

О. А. Юшков

Байкальский государственный университет, 2. Иркутск, Российская Федерация

\title{
ОСОБЕННОСТИ УПРАВЛЕНИЯ РИСКАМИ НА МИКРОУРОВНЕ В РЕАЛЬНОМ СЕКТОРЕ ЭКОНОМИКИ
}

\begin{abstract}
АНнОтАЦия. В экономической литературе и на практике на сегодняшний момент существует много разночтений и противоречивых подходов к понятию риска, к риск-менеджменту и оценке уровня неопределенности. Одни авторы опираются на использование достижений экономико-математического моделирования, другие отказываются от практики использования теоретических абстракций при построении математических моделей в пользу реального менеджмента. Данное противоречие, по мнению авторов данной статьи, нивелируется, если разграничить сферы применения данных подходов. Неоднозначная и сложная ситуация складывается в реальном секторе экономики, который, в силу своих сложных субъектно-объектных отношений, определяет специфику процесса управления рисками в этом секторе. Процессы построения эффективной модели риск-менеджмента довольно сильно зависят от качественно выстроенной системы менеджмента в компании в целом. Высокую роль играют не только компетенции персонала при исполнении своих функций, но и организационно-структурная схема взаимодействия внутри компании, успешное диагностирование ситуации в самой компании и основных тенденций во внешней среде, особенно на мезо уровне. Кроме этого, эффективность риск-менеджмента определяется способностью и скоростью адаптации внутренней среды к внешним условиям и организацией взаимовыгодного взаимодействия с контрагентами и иными представителями мезо среды.

кЛючЕВЫЕ слОВА. Риск-менеджмент; управление рисками; неопределенность; реальный сектор экономики; экономикой-математическое моделирование.

ИНФОРМАЦИЯ О СТАТЬЕ. Дата поступления 11 апреля 2017 г.; дата принятия к печати 28 апреля 2017 г.; дата онлайн-размещения 22 июня 2017 г.
\end{abstract}

E. V. Kolesnikova Baikal State University, Irkutsk, Russian Federation

O. A. Yushkov Baikal State University, Irkutsk, Russian Federation

\section{FEATURES OF RISK MANAGEMENT AT THE MICRO LEVEL IN REAL SECTOR OF ECONOMY}

\begin{abstract}
In economic literature and in practice there are many variant readings and controversial approaches to the concept of risk, to risk management and assessment of uncertainty level. Some scientists draw upon using achievements of economic and mathematical modeling, while the others reject the practice of using theoretical abstractions in building mathematical models in favor of real management. An ambiguous and complicated situation is being developed in the real economic sector which, due to its complex subject-object relationship, determines the specificity of the risk management process in this sector. The processes of building an effective system of risk management in the company depend quite heavily on the effectively built management system in the company. Of great significance are not only the personnel's competence in performing their functions but also the organizational and
\end{abstract}

(C) E. В. Колесникова, О. А. Юшков, 2017

\section{Baikal Research Journal}


structural scheme of the interaction in the company, the successful diagnosing of the situation in the company and main trends in the external environment, especially at the mezo-level. Besides, the risk management effectiveness is determined by the ability and speed of the internal environment adaptation to external conditions and organization of mutually profitable interaction with counter agents and other representatives of the mezo-environment. The role played not only by the competence of personnel in the performance of their activities, but also by the successful diagnosis of the situation not only within the company, but also the degree and speed of reaction of the influence of external counterparties on the ongoing changes in the company and the economy of the country and the region as a whole.

KEYWORDS. Risk management; uncertainty; real sector of economy; economic-mathematical modeling.

ARTICLE INFO. Received April 11, 2017; accepted April 28, 2017; available online June 22, 2017.

Риск-менеджмент в реальном секторе экономики в настоящее время является особенно актуальным объектом исследования в силу возросшего числа факторов, влияющих на бизнес, в том числе факторов не экономического характера, а также в силу интенсивности и разнонаправленности самого влияния. Не последнюю роль играют процессы глобализации.

Между тем, до тех пор, пока мы не определимся с объектом управления, мы не можем эффективно им управлять. В этой связи встает ключевой вопрос о том, что мы понимаем под риском. Рамки настоящей статьи не позволяют нам остановиться на подробном анализе всех подходов к данному понятию. Хотя обозначить их возможно и целесообразно.

Риск, традиционно, в литературе определяется как количественная и качественная оценка вероятности неудачи, не достижения желаемого результата или отклонения от поставленной цели. Рисковое событие может произойти вследствие неопределенности, с некоторой вероятностью, и влечет за собой разный характер последствий. Причем некоторые исследователи полагают, что если последствия могут носить, как положительный, так и отрицательный характер, то и значение риска, соответственно, может быть положительным, отрицательным и даже нулевым. В таком подходе, на наш взгляд, отсутствует логика. То есть, если вероятность рискового события и размер возможных в следствие него потерь ниже вероятности благоприятного стечения обстоятельств и размера положительного в следствие этого результата, то риск считается положительным. То есть, если риск меньше эффекта. Порой, всякий возможный положительный исход событий в условиях неопределенности рассматривается, как положительный риск, а отрицательный исход, соответственно, как риск отрицательный. Это похоже на «Соломоново» решение.

Традиционный риск-менеджмент, описываемый в литературе, опирается на математические методы и модели (Л. Канторович, Т. Кумпанс) [1, с. 211$].$ Приверженцы этой теории избегают понятия определенности и не используют ее в своей теории, и строят ее на предположениях детерминированности развития экономической системы [2; 3$]$.

В противоположность этой школе, возникла и другая. Ее главными представителями стали Ф. Котлер и П. Друкер, которые продвигали идеи дедуктивных конструкций и отказывались абстрагироваться от окружающей реальности и переходить к изучению экономики с помощью использования исключительно математических моделей [4; 5].

Мы считаем второй подход более обоснованным, так как неопределенность является лишь источником вероятного рискового события, а вероятность, может

\section{Baikal Research Journal}


характеризовать не только степень рискованности, но и благоприятности. Поэтому риск не следует отождествлять с понятиями неопределенность и вероятность.

По нашему мнению, модель управления рисками, сама по себе, ни на уровне хозяйствующего субъекта в целом, ни на уровне процессов, происходящих на предприятиях, не может однозначно опираться на математические модели и математическую оценку вероятности возникновения какого-либо событий. Это невозможно в силу того, что на этом уровне не всегда проявляют себя закономерности, действующие в рамках теории больших чисел.

Риск для каждого участника производственно-хозяйственных отношений субъективен, следовательно, для наиболее эффективной организации «риск-менеджмента" необходимо вовлечение как можно большего количества субъектов этих отношений и максимальное согласование их интересов.

Нам импонирует представление о риске, как вероятностной категории, которая отражает характер и масштаб потерь при некоторых событиях и обстоятельствах, возможных в будущем. То есть риск - это количественно измеряемая величина возможного ущерба.

Действительно, покупатель лотерейного билета имеет высокую вероятность ущерба (риска), но сам ущерб (риск) определяется лишь ценой билета. Так, как покупатель билета не может влиять на проведение тиража и не его итоги, то неопределенность результатов тиража очень высока. Но покупатель готов потерять небольшую сумму денег (идти на риск) в надежде на выигрыш, который многократно может превышать стоимость билета. Конечно, мы не учитываем альтернативные издержки покупателя, но они, в данном случае, будут не высоки, так как стоимость билета тоже не велика.

Но на данную ситуацию можно посмотреть и, с другой стороны. Отказавшись от покупки лотерейного билета, человек рискует не получить выигрыш. Эффектом в данном случае будет выступать экономия на стоимости билета. Значит риск можно рассматривать, как явный и не явный. То есть мы готовы оказаться (рискнуть) от того что имеем ради того значительно большего, что можем получить в будущем. Либо отказываемся от некоторого возможного блага в будущем, сохраняя то, что имеем в настоящем. Но, в любом случае, риск - это некоторый ущерб, явный или не явный.

В разных сферах проявления характер риска (потерь) разный. В экономической сфере наиболее измеряемые потери носят финансовый и имущественный характер. Мы убеждены, что рисками, как и эффектами управлять возможно лишь опосредованно. Дело в том, что управлению поддаются процессы, люди и финансовые потоки, а качество управления ими способствует минимизации риска и максимизации эффекта. Более того, имеет смысл вести речь об управлении рискованностью и эффективностью, поскольку риск и эффект являются понятиями абсолютными, а поэтому не могут характеризовать эффективность самого управления, которое предполагает определенные затраты. Порой затраты на мероприятия, направленные на минимизацию вероятности возникновения рискового события (события, при котором возникает риск, как потеря чего-либо) могут оказаться выше самого возможного риска. Рискованность характеризуется вероятностью потерь и их величиной. При этом, мы полагаем, что перспективная эффективность и рискованность поддается количественному измерению условно, так как причинно-следственные связи между техническими (технологическими), социальными, экономическими и иными факторами не всегда поддаются жесткой математизации.

Конечно, теория управления рисками достаточно развита и, часто, опирается на теорию вероятностей. Однако теория вероятностей применима лишь в рамках действия теории больших чисел и в рамках условно конечного количества одно-

\section{Baikal Research Journal}


родных событий. Поэтому она эффективно может применяться на уровне экономики отрасли, региональной, национальной и мировой экономики.

На финансовых рынках для инвестора, так же, актуальным является, именно, математическая модель управления рисками, основанная на вероятностном подходе, так как сам инвестор не может влиять на доходность каждого конкретного финансового инструмента, но может количественно оценить соотношение между доходностью и рискованностью финансового актива. Вероятностный подход, в данном случае возможен в силу наличия большого количества предшествующих событий относительно колебаний курсов отдельно взятого финансового инструмента, их взаимосвязи с курсами других ценных бумаг и тенденций на фондовых рынках, которые, в значительной степени, зависят от макроэкономических факторов. Кроме этого, в данной сфере, рисками (как и рискованностью) можно управлять за счет формирования портфеля бумаг, который может иметь определенный уровень диверсификации, консервативности (агрессивности) и ликвидности. Основным объектом управления являются финансовые потоки.

В реальном секторе предпринимательства, в сравнении с финансовым, характер управленческих решений и спектр зон ответственности, в рамках которых эти решения принимаются значительно шире.

На уровне отдельно взятой компании количественно измерить вероятность возникновения того или иного рискового события, которое может привести к потерям, оценить не всегда представляется возможным. И уж совсем невозможно оценить вероятность совокупности событий, их взаимного влияния на общую величину риска. Правда, по ряду причин, исключение могут составлять очень крупные, в том числе транснациональные компании и корпорации.

Вероятностный подход к управлению рисками заставляет погружаться в математические расчеты и отвлекаться от реальных событий и процессов, которые требуют изучения, принятия определенных управленческих решений и усилий для их выполнения. Более того, в этой сфере может быть не возможной, а порой, и опасной опора на количественную оценку вероятности возникновения рискового события, так как причинно-следственные связи имеют достаточно широкий диапазон.

Поэтому математический аппарат может использоваться в рамках существующих экономических методик лишь как инструмент оценки качества управленческих решений, которые принимаются для предотвращения возможных рисковых событий или для уменьшения ущерба в случае, если рисковое событие избежать не удается.

Мы полагаем, что последовательность проявления результатов управленческих решений в хозяйствующих субъектах имеет вид, который представлен на рисунке.

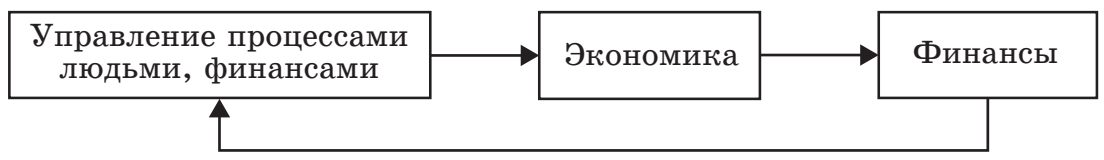

Последовательность проявления результатов управленческих решений в хозяйствующих субъектах

В связи с причинно-следственными связями, отраженными на рисунке, в рамках отдельно взятой компании, мы предлагаем использовать не вероятностный, а «возможностный» подход.

Отличие заключается в том, что следует делать акцент не на количественной оценке вероятности возникновения рискового события в той или иной сфере проявления, а на оценке возможности избежать или предотвратить рисковое событие

\section{Baikal Research Journal}


или на уменьшении самого риска, если события невозможно избежать. Но полностью исключить неопределенность невозможно, значит, вероятность рискового события сохраняется.

Если в экономической среде неопределенность - это более объективное явление, чем определенность, то вероятность возникновения рискового события достаточно оценивать на уровне высокой, средней и низкой. При этом важно разделить риски на некоторые группы. Например, на инвестиционные, коммерческие, производственные и риски финансовых операций. Уровень инвестиционных рисков зависит от уровня ликвидности объектов инвестирования, продолжительности инвестиционного периода и т. д. Уровень коммерческих рисков (риски недопродаж или недополучения выручки) зависит от отрасли и вида бизнеса и, следовательно, от качества комплекса маркетинга. Производственные риски (риски недопроизводства и удорожания себестоимости) зависят от качества организации труда и производства на каждом конкретном производстве.

Следовательно, управление рисками и рискованностью должно сводиться к построению некоторой модели управления, в которой четко определяются качественные характеристики элементов данной модели и характер связей между элементами.

Поэтому, эффективная модель должна быть такой, которая позволяет минимизировать или даже полностью устранять вероятность возникновения тех или иных событий, которые могут привести к рискам (потерям) либо минимизировать сами риски, в условиях, когда на рисковые события влиять не удается. Построение такой модели предполагает дополнительные затраты, разовые и текущие. Разовые затраты могут быть связаны с созданием определенной организационной или структурной модели управления, с формированием технологической базы, с разработкой комплекса маркетинга и т. д. Текущие, например, - с формой и размером оплаты труда работников заданного уровня образования и квалификации, с качеством сырья и материалов и т. д. [6, с. 146].

Таким образом, мы приходим к выводу о построении не математической модели управления рисками, а организационно - структурной, которая определяет поведенческие реакции менеджмента на возможные рисковые события. Математическая модель управления рисками (рискованностью) может использоваться для анализа возможных событий, на которые компания влиять не может [7, с. 81]. Например, относительно отраслевых, национальных и мировых экономических, политических и иных тенденций, которые могут оказать влияние на деятельность компании в тех или иных сферах предпринимательства с определенной вероятностью. Уже в рамках этих тенденций внутрифирменная модель управления рискованностью должна «работать» либо в направлении максимизации эффекта и эффективности (в условиях благоприятных), либо в направлении минимизации риска и рискованности (в условиях неблагоприятных). Следовательно, эффективная модель должна строиться на принципах максимина. Именно такой подход может явиться критерием әффективности модели управления рисками в компании. Иными словами, количественный учет вероятности тех или иных неблагоприятных для компании событий, на которые она влиять не может, позволяет принять решение о готовности или неготовности руководства компании идти на возможные потери ради возможности получения определенных дополнительных эффектов. При этом встает вопрос о цене риска. Чем выше цена риска, тем меньше должна быть вероятность рискового события. При этом, цена риска определяется не масштабом потерь, а их значимостью для компании с точки зрения отрицательных последствий. Именно цена риска, а не вероятность его возникновения, отражает степень оправданности решения идти на риск.

\section{Baikal Research Journal}

электронный научный журнал Байкальского государственного университета 
Неблагоприятные для компании события, возможность которых она может минимизировать или полностью устранить за счет структурных, организационных и поведенческих внутрифирменных механизмов, следует рассматривать как управляемые объекты влияния. Следовательно, чем выше масштаб, и, тем более, цена вероятного риска, тем больше затрат и управленческих усилий требуется на снижение вероятности возникновения рискового события.

В этом случае управление рисками должно осуществляться в обратной последовательности по отношению к той, что отражена на рисунке. С учетом факторов риска, могущих повлиять на результирующие финансовые показатели деятельности компании и зон их проявления (на входе в производственно - хозяйственную систему, в самой системе и ее звеньях и на выходе из системы), в каждой из этих зон выявляются управляемые объекты влияния, на которые следует ориентировать эффективный механизм управления рисками и рискованности в компании. Основу данного механизма должны составлять организационные, структурные и поведенческие элементы [8, с. 122].

Данный посыл можно проиллюстрировать довольно простым примером. Допустим, есть вероятность уменьшения компанией объемов продаж в будущем. Это, однозначно, окажет отрицательное влияние на выручку, удельные текущие издержки и прибыль [9, с. 179]. Необходимо оценивать не математическую вероятность рискового события, а выявлять его причины. Это может быть недоступность товара в будущем для некоторых целевых потребителей по цене в силу снижения покупательной способности, будущая тенденция к снижению цены в силу насыщение конкурентами грынка товарами данного рода или неудовлетворенность потребителя в перспективе качеством товара, и т. д. Значит необходимо выявить причины рискового события.

Если причиной является ценовой фактор, то для компании, в себестоимости продукции которой доля постоянных затрат высока, даже не значительное изменение продаж может привести к критическим, и даже катастрофическим, потерям. В условиях, если спрос на товар эластичен, то сохранить продажи возможно за счет снижения цены и пойти на риск на уровне допустимого. Соотнеся необходимые инвестиции на совершенствование технологического процесса с целью снижения текущих затрат, можно принять решение о финансировании инвестиций. В условиях, когда товар относится к категории товаров, обладающих коротким периодом морального устаревания, данное решение может оказаться не верным.

Если на объемы продаж будут влиять возрастающие требования потребителей к качеству продукции, то без инвестиций в совершенствование продукции и технологического процесса не обойтись. Определение параметров качества, которые предпочтет потребитель в будущем, возлагается на маркетологов, отвечающих за разработку маркетингового комплекса, Способы достижения заданных свойств товар - на разработчиков, способы минимизации издержек и обеспечения требуемого объема производства - на технологов и производственников, обеспечение оптимальных источников финансирования проектных решений на финансистов.

Пример, конечно, достаточно утрированный, но важным моментом во всех суждениях, отраженных в данной статье является то, что управление рисками сводится к внутрифирменному взаимодействию функциональных сфер бизнеса по трем основным вариантам принятия решений в условиях неопределенности: действию, бездействию и противодействию. Действие направлено на использование возможностей, чтобы не явные эффекты сделать явными, бездействие в условиях, когда затраты на устранение рискового события выше величины риска, а противодействие ориентировано на минимизацию вероятности возникновения

\section{Baikal Research Journal}

электронный научный журнал Байкальского государственного университета 
рискового события или на минимизацию самого риска, если затраты на это ниже предотвращаемого риска.

Поэтому организационные, структурные и поведенческие элементы, которые составляют ключевое содержание модели управления рисками, должны быть органично взаимосвязаны и не должны вступать в противоречие [10, с. 54]. Все внутрифирменные элементы модели при этом должны взаимодействовать с элементами мезо уровня компании (табл.).

Элеленты модели управления рисками в колпании

\begin{tabular}{|l|c|c|c|}
\hline \multirow{2}{*}{ Зоны рисков } & \multicolumn{3}{|c|}{ Элементы модели } \\
\cline { 2 - 4 } & Организация & Структура & Поведение \\
\hline Инвестиционная сфера & + & + & + \\
\hline Производственная сфера & + & + & + \\
\hline Сбытовая сфера & + & + & + \\
\hline
\end{tabular}

Такой подход во многих случаях позволяет нивелировать элемент случайности в управлении рисками на уровне хозяйствующего субъекта.

\section{Список использованной литературы}

1. Канторович Л. В. Экономический расчет наилучшего использования ресурсов / Л. В. Канторович. - М. : Изд-во Акад. наук СССР, 1959. - 344 с.

2. Панфилова Э. А. Понятие риска: многообразие подходов и определений / Э. А. Панфилова // Теория и практика общественного развития. - 2010. - № 4. - С. 30-34.

3. Панягина А. Е. Подходы к пониманию и классификации рисков / А. Е. Панягина // Современная экономика: проблемы, тенденции, перспективы. - 2012. - № 6. - С. 1-10.

4. Гримашевич О. Н. Формирование системы управления рисками промышленного предприятия / О. Н. Гримашевич // Вестник Саратовского государственного социальноэкономического университета. - 2011. - № 2. - С. 72-76.

5. Пилюк С. С. Управление рисками экспорто-ориентированных предприятий на зарубежных рынках / С. С. Пилюк, В. А. Журавлев, Ю. А. Ивсин // In Situ. - 2015. № $1 .-$ C. $48-54$.

6. Иванов А. А. Риск-менеджмент / А. А. Иванов, С. А. Олейников, С. А. Бочаров. М. : Евраз. открыт. ин-т, 2008. - 193 с.

7. Самохвалова М. Г. Методы управления хозяйственным риском / М. Г. Самохвалова // Известия Иркутской государственной экономической академии. - 2007. № 3 (53). - C. 80-83.

8. Субботницкий Д. Ю. Риск и неопределенность: бесконечный путь к недостижимой истине / Д. Ю. Субботницкий // Известия Российского государственного педагогического университета им. А.И. Герцена. - 2009. - Т. 97. - С. 121-125.

9. Риск-менеджмент инвестиционного проекта / под ред. М. В. Грачевой, А. Б. Секерина. - М. : Юнити-Дана, 2009. - 544 с.

10. Каранина Е. В. Оптимизация процесса систематизации и оценки рисков предприятия в кризисных условиях / Е. В. Каранина // Вестник Пермского университета. 2010. - № 4 (7). - C. 50-63.

\section{References}

1. Kantorovich L. V. Ekonomicheskii raschet nailuchshego ispol'zovaniya resursov [Economic calculation of the best resource use]. Moscow, Academy of Sciences of the USSR Publ., 1959. $344 \mathrm{p}$.

2. Panfilova E. A. Concept of risk: variety of approaches and definitions. Teoriya i praktika obshchestvennogo razvitiya $=$ Theory and Practice of Social Development, 2010, no. 4, pp. 30-34. (In Russian).

3. Panyagina A. E. Approaches to understanding and classification of risks. Sovremennaya ekonomika: problemy, tendentsii, perspektivy = Modern Economics: Problems, Trends, Prospects, 2012, no. 6, pp. 1-10. (In Russian).

\section{Baikal Research Journal}


4. Grimashevich O. N. Risk management system in industrial enterprises. Vestnik Saratovskogo gosudarstvennogo sotsial'no-ekonomicheskogo universiteta $=$ Bulletin of Saratov State Socio-Economic University, 2011, no. 2, pp. 72-76. (In Russian).

5. Pilyuk S. S., Zhuravlev V. A., Ivsin Yu. A. Risk management in export-oriented companies on foreign markets. In Situ, 2015, no. 1, pp. 48-54. (In Russian).

6. Ivanov A. A., Oleyinikov S. A., Bocharov S. A. Risk-menedzhment [Risk management]. Moscow, Eurasian Open Institute Publ., 2008. 193 p.

7. Samokhvalova M. G. Methods of managing economic risks. Izvestiya Irkutskoi gosudarstvennoi ekonomicheskoi akademii = Bulletin of Irkutsk State Economics Academy, 2007, no. 3 (53), pp. 80-83. (In Russian).

8. Subbotnitsky D. Risk and uncertainty: the infinite way to the unattainable truth. Izvestiya Rossiiskogo gosudarstvennogo pedagogicheskogo universiteta im. A. I. Gertsena = A. I. Herzen State Pedagogical University Bulletin, 2009, vol. 97, pp. 121-125. (In Russian).

9. Gracheva M. V., Sekerin A. B. (eds). Risk-menedzhment investitsionnogo proekta [Risk-management of investment projects]. Moscow, Yuniti-Dana Publ., 2009. 544 p.

10. Karanina E. V. Optimizing the process of systematization and assessment of company's risks in terms of crisis. Vestnik Permskogo universitet $=$ Perm University Herald, 2010, no. 4 (7), pp. 50-63. (In Russian).

\section{Информация об авторах}

Колесникова Елена Валерьевна - аспирант, кафедра экономики предприятия и предпринимательской деятельности, Байкальский государственный университет, 664003, г. Иркутск, ул. Ленина, 11; e-mail: ulyanenko.helen@mail.ru.

Юшков Олег Александрович - старший преподаватель, кафедра экономики предприятия и предпринимательской деятельности, Байкальский государственный университет, 664003, г. Иркутск, ул. Ленина, 11; e-mail: oay63@mail.ru.

\section{Authors}

Elena V. Kolesnikova - PhD Student, Chair of Business Economy and Entrepreneurial Activity, Baikal State University, 11 Lenin St., 664003, Irkutsk, Russian Federation; e-mail: ulyanenko.helen@mail.ru.

Oleg A. Yuskov - Senior Lecture, Chair of Business Economy and Entrepreneurial Activity, Baikal State University, 11 Lenin St., 664003, Irkutsk, Russian Federation; e-mail: oay63@mail.ru.

\section{Библиографическое описание статьи}

Колесникова Е. В. Особенности управления рисками на микроуровне в реальном секторе экономики / Е. В. Колесникова, О. А. Юшков // Baikal Research Journal. — 2017. T. 8, № 2. - DOI: 10.17150/2411-6262.2017.8(2).30.

\section{Reference to article}

Kolesnikova E. V., Yushkov O. A. Features of risk management at the micro level in real sector of economy. Baikal Research Journal, 2017, vol. 8, no. 2. DOI: 10.17150/24116262.2017.8(2).30. (In Russian).

\section{Baikal Research Journal}

